\title{
Features of the manifestation of lunar-solar tides in the electromagnetic parameters of the active fault zones of the Tien Shan
}

\author{
Elena Bataleva \\ Research Station of the Russian Academy of Sciences in Bishkek, 720049, Bishkek, \\ Kyrgyzstan
}

\begin{abstract}
The results of monitoring studies of the electromagnetic parameters of active fault structures on the territory of the Bishkek geodynamic proving ground are shown. The temporal variations of the electromagnetic field on several active faults of the Earth's crust, characterized by different tectonic activity, are analyzed in comparison with the variations of lunar-solar tides. It was found that in the overwhelming majority of cases, the correlation dependences are most clearly manifested in changes in the real and imaginary parts of additional impedances than the main ones. Analysis of the cross-correlation function indicates that the reason for the change in the parameters of the electromagnetic field can be lunar-solar tides.
\end{abstract}

\section{Introduction}

The study of lunar-solar tidal effects on the Earth's surface is one of the most important areas of research in the physics of the Earth. Of particular interest among the phenomena accompanying lunar-solar tidal deformations, in our opinion, their potential relationship with the electrical conductivity of rocks, which may be due to the fluid regime of the Earth's crust or the preparation of seismic events [1-5]. One of the first interesting results of assessing the influence of lunar-solar tides on the electrical conductivity of the Earth's crust was obtained in Japan in the middle of the last century when solving problems of electromagnetic monitoring [6]. When conducting monitoring studies in such seismically active regions as the Tien Shan, it is necessary to have an idea of the patterns of behavior of active fault zones, including as a fluid-saturated medium under the influence of seismic events. The method of magnetotelluric sounding (MTS), one of the leading methods of electromagnetic monitoring of the Bishkek geodynamic proving ground (BGPG), allows monitoring hidden fault structures which do not appear in the sedimentary cover, but are zones of increased fracturing and fluid permeability of the basement and are manifested by anomalies in geophysical fields.

Currently, on the territory of the BGPG (Fig. 1), the main experimental studies are aimed at studying the zones of increased fracturing and fluid saturation. The increased interest in the study of fault zones is associated with their important role in the formation of

\footnotetext{
* Corresponding author: elena.bataleva@gmail.com
} 
the stress-strain state of the Earth's crust in seismically active regions, the regime of underground fluids, as well as the processes of mass, heat and energy exchange in the zones of their dynamic influence [7-9]. These factors also determine a new stage in the development of studies of the nature of deformation processes in the Earth's crust of the Bishkek proving ground. The development of monitoring technologies in the geosciences, the widespread use of GPS observations, broadband digital seismic stations, and modern electrical survey equipment have led to the emergence of a huge amount of experimental material that needs adequate interpretation.

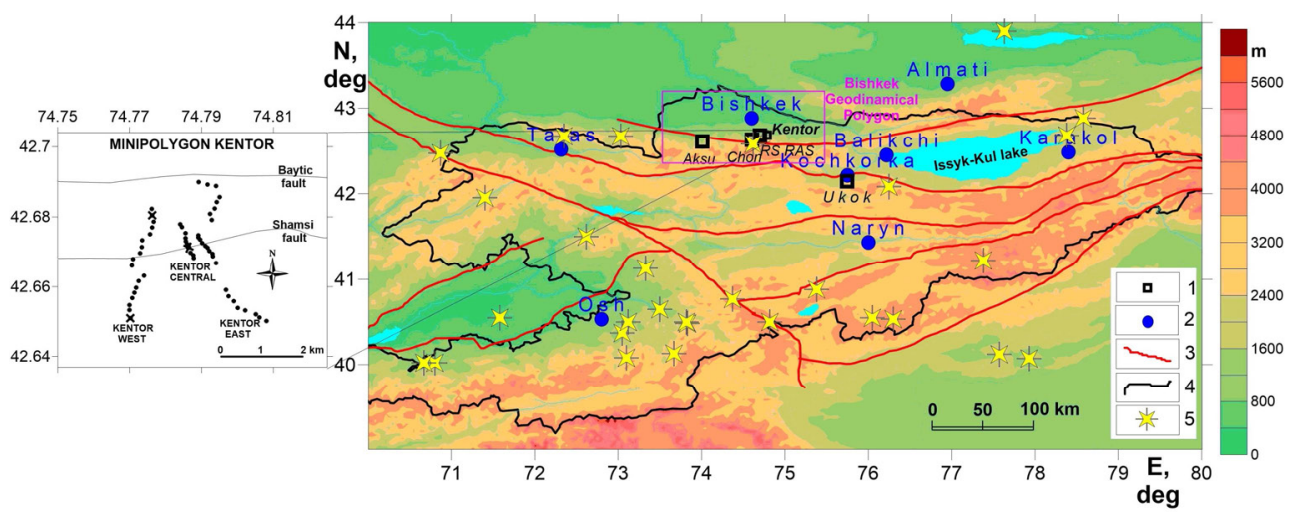

Fig. 1. Location map stationary points of the MT-monitoring, point DMT Ukok, minipoligon Kentor (insert), performed on the territory of the Central Tien Shan: 1 - stationary points of MT-monitoring and point DMT Ukok; 2 - main settlements; 3 - regional faults; 4 - border of Kyrgyzstan; 5 - seismic events that occurred during the registration at the point of DMT Ukok.

\section{Technique of magnetotelluric monitoring of active fault zones of the Tien Shan}

To detect and study fracture zones, both the analysis of traditional electromagnetic parameters (apparent resistivity, impedance phase, etc.) and new ones - anisotropic changes in the electrical conductivity of the geological medium are used, the assessment of which is carried out on the basis of methodological approaches specially developed over the past decade for carrying out monitoring studies. The geological environment, in general, and the fault structure, in particular, is subject to periodic effects of lunar-solar tides, as a result of which its parameters (electrical conductivity, fluid saturation, elasticity, etc.) also periodically change. In turn, variations in these parameters reflect the dynamics of geophysical fields in the upper layers of the crust. The results of experimental observations in the zones of dynamic influence of active faults are presented both in the form of timefrequency series (Fig. 2) and in the form of correlation diagrams (Fig. 3).

Time-frequency series (TFS) are a form of visualization of the variability of the structure of the measured components of the electromagnetic field (logarithm of the sounding period) when the coordinate system is rotated by a certain angle (in degrees) [10]. Figure 3 shows TFS with a step of $15(30)^{\circ}$ in azimuth, where variations in apparent resistivity $(\rho c)$ are plotted, i.e., the difference between the average and current values along the considered azimuth. The formation of the indicated time series of variations in MT parameters was carried out according to the following scheme: 1) processing of MT records using the SSMT-2000 program (Phoenix Geophysics) and calculating the values of the frequency dependences of the impedance tensor for disjoint hourly or two-hour intervals in the range of periods from 0.01 to $100 \mathrm{~s}$ ( the total duration of the MT-recording at security 
observation points is at least 48 hours); 2) recalculation of the impedance tensor values on the azimuth grid after 15 degrees. As a result, the resulting array of experimental data includes the values of the impedance tensor components for at least 48 intervals of one hour each in the range of periods from 0.01 to $100 \mathrm{~s}$ and the range of azimuths from $0^{\circ}$ to $360^{\circ}$. The temporal dynamics of variations in MT parameters calculated for the entire set of periods and azimuths is of greatest interest in this work. The results of the correlation analysis are presented in the form of polar diagrams (Fig. 3), where the azimuth axis corresponds to the recalculation angle of the impedance tensor components, the root of the MT sounding period is plotted along the radius axis, and the values of the correlation coefficients are color-coded.

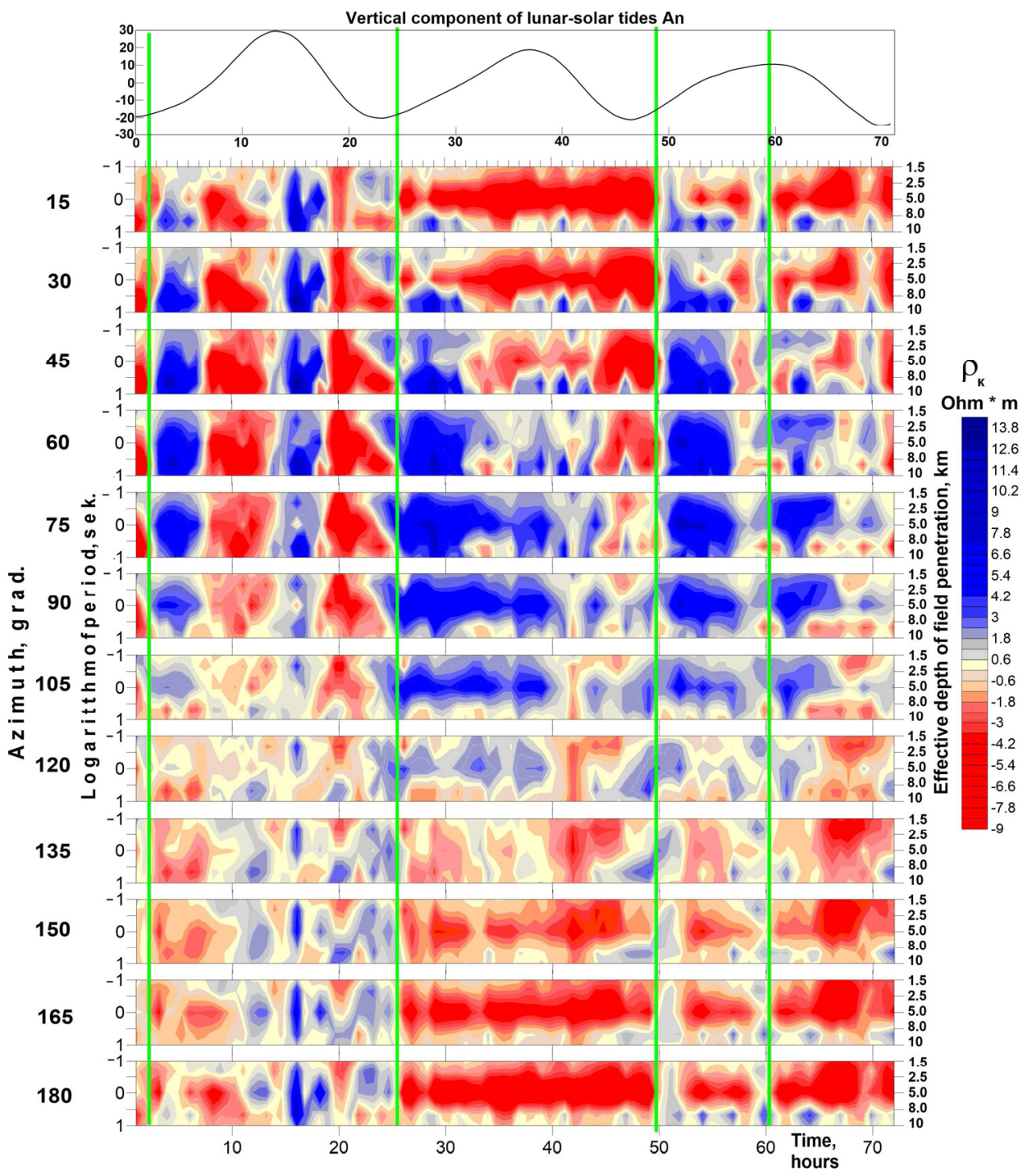

Fig. 2. Comparison of variations in apparent electrical resistivity (Kambarata point) with lunar-solar tidal effects and seismicity distribution. In the upper part is the vertical component of the lunar-solar tides An. Below - apparent resistivity in different azimuths. Green lines - earthquakes that occurred during the experiment. 

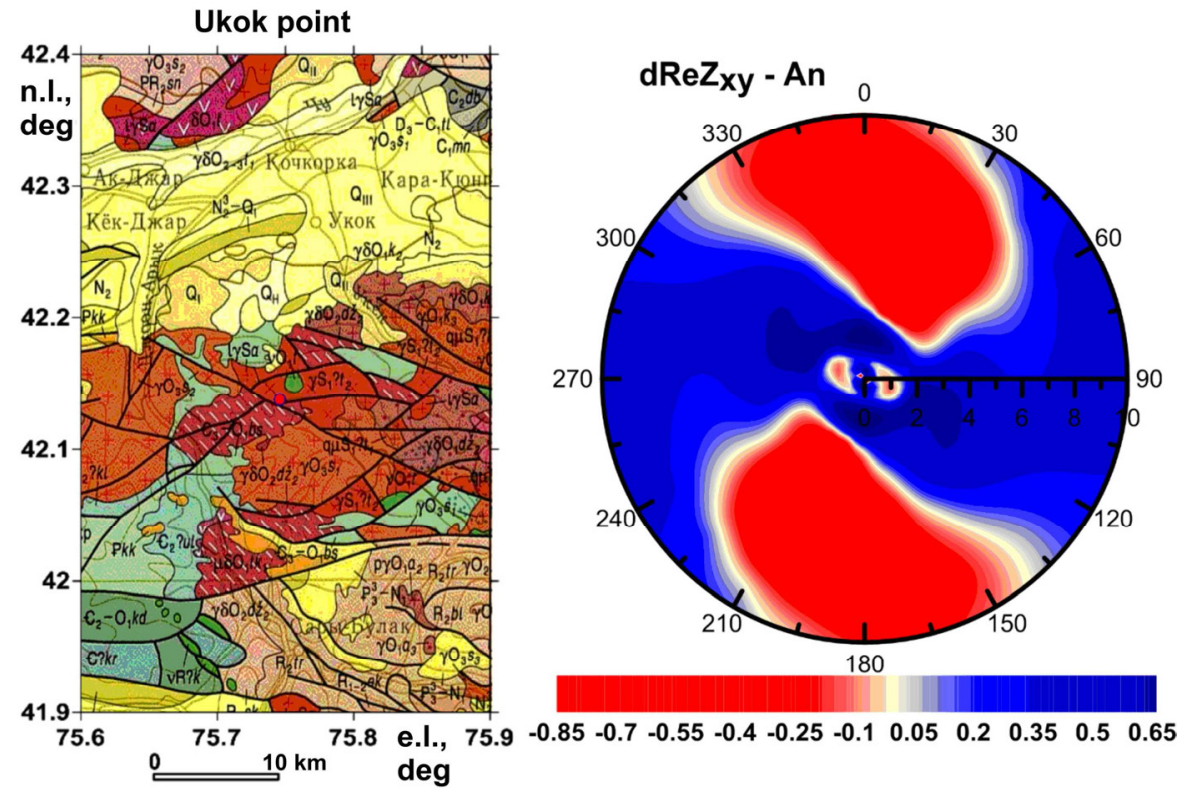

Fig. 3. Panel on the left - a fragment of the geological map near the location of the Ukok MTmonitoring regime point; the panel on the right - the distribution of the correlation coefficient (the main impedance Zxy and the vertical component of tidal forces An) in polar coordinates, where the $\sqrt{T}_{\mathrm{T}}$ (T-period) is shown along the radius axis. Color and contour lines show the values of the correlation coefficient.

To calculate the lunar-solar tides, we used the TIDE_3.exe program. As a result of the program operation, in the TIDEALL.txt file (lunar-diurnal variations) for each hour, four values for the tide curve are obtained: the eastern component $(\mathrm{Au})$, the northern component $(\mathrm{AV})$, the vertical component (An), and the modulus of the total vector (A). Basically, semidiurnal and diurnal tidal waves are calculated for the Earth with the ocean and for the solid Earth (excluding the ocean). However, the calculated curve will differ from the observed one due to many factors, such as the type, thickness and structure of the earth's crust. Discrepancies between the observed field and the calculated curve can be removed using the "GS-project program for primary processing of the data of registration of the gravity field". The program is designed for primary processing of the gravity field registration data with a high-precision Scintrex CG-5 gravimeter installed in an adit on the territory of the Research Station RAS, which is used to study the Earth's tidal deformations experimentally. The program provides the ability to link the file of the calculated vertical component of the lunar-solar tide to the gravimeter record and divide the measured gravitational field into tidal and non-tidal components.

\section{Results of experimental observations at magnetotelluric monitoring points}

A comparative analysis of the dynamics of the behavior of the parameters of the electromagnetic field and lunar-solar tides was carried out on the basis of materials obtained during experimental observations at the regime monitoring station of sounding in the Ukok-2 Kochkor basin (South Kochkor fault) (Fig. 3), stationary observation points Ak-Suu (Issyk-Ata fault) and Chon-Kurchak (Chon-Kurchak fault) (Figs. 4,5) and on the monitoring profiles of the Kentor mini-polygon (Shamsi-Tyundyuk fault) (Fig. 6). 

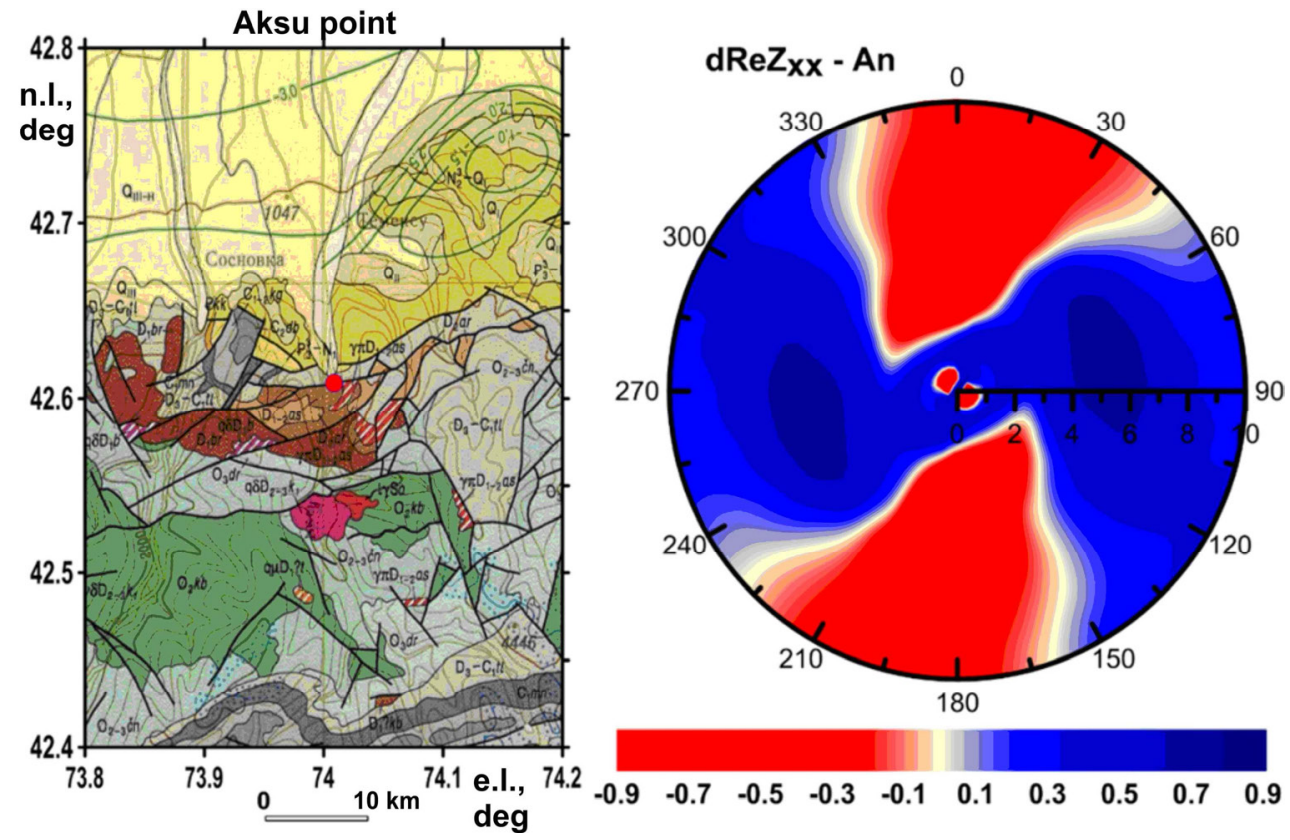

Fig. 4. Panel on the left - a fragment of the geological map near the location of the stationary MTmonitoring Aksu point; panel on the right - distribution of the correlation coefficient (additional impedance Zxx and vertical component of tidal forces An) in polar coordinates, where the $\sqrt{T}_{\mathrm{T}}(\mathrm{T}$ period) is shown along the radial axis. Color and contour lines show the values of the correlation coefficient.

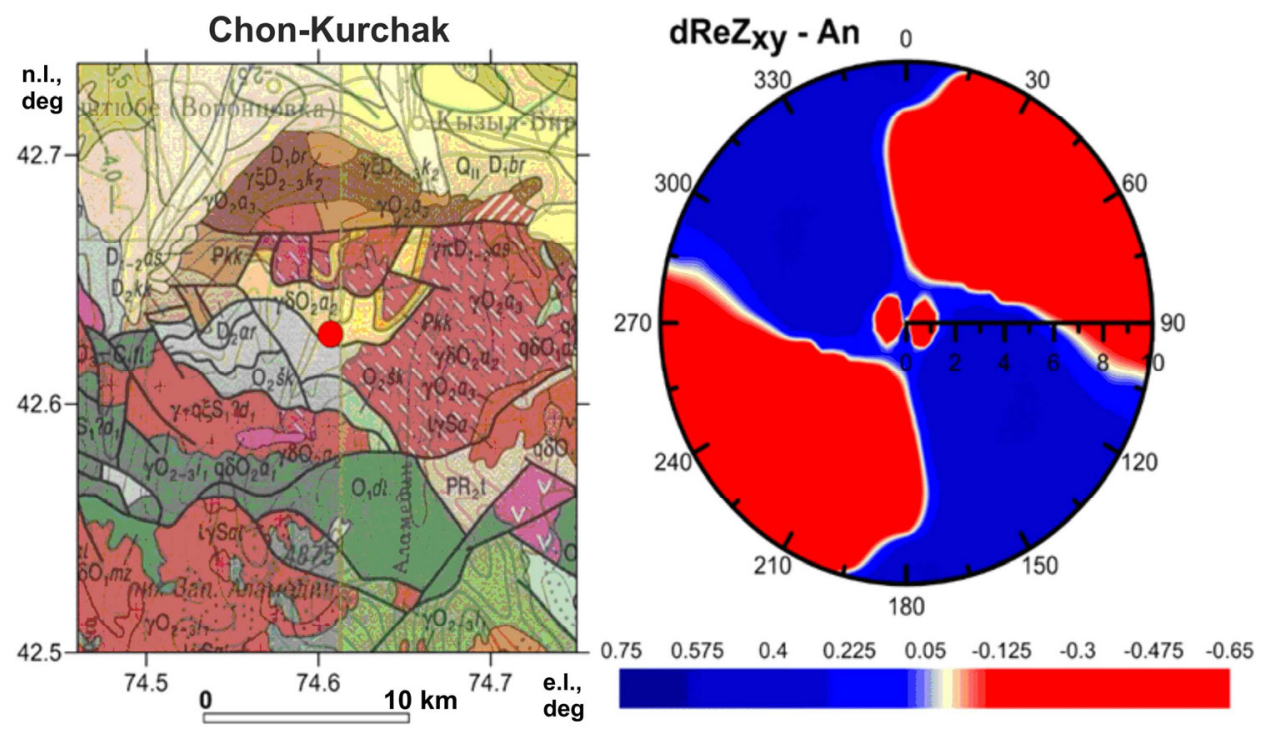

Fig.5. Panel on the left - a fragment of the geological map near the location of the stationary MTmonitoring station Chon-Kurchak; the panel on the right - the distribution of the correlation coefficient (additional impedance Zxy and the vertical component of tidal forces An) in polar coordinates, where the $\sqrt{\mathrm{T}}$ (T-period) is shown along the radius axis. Color and contour lines show the values of the correlation coefficient. 


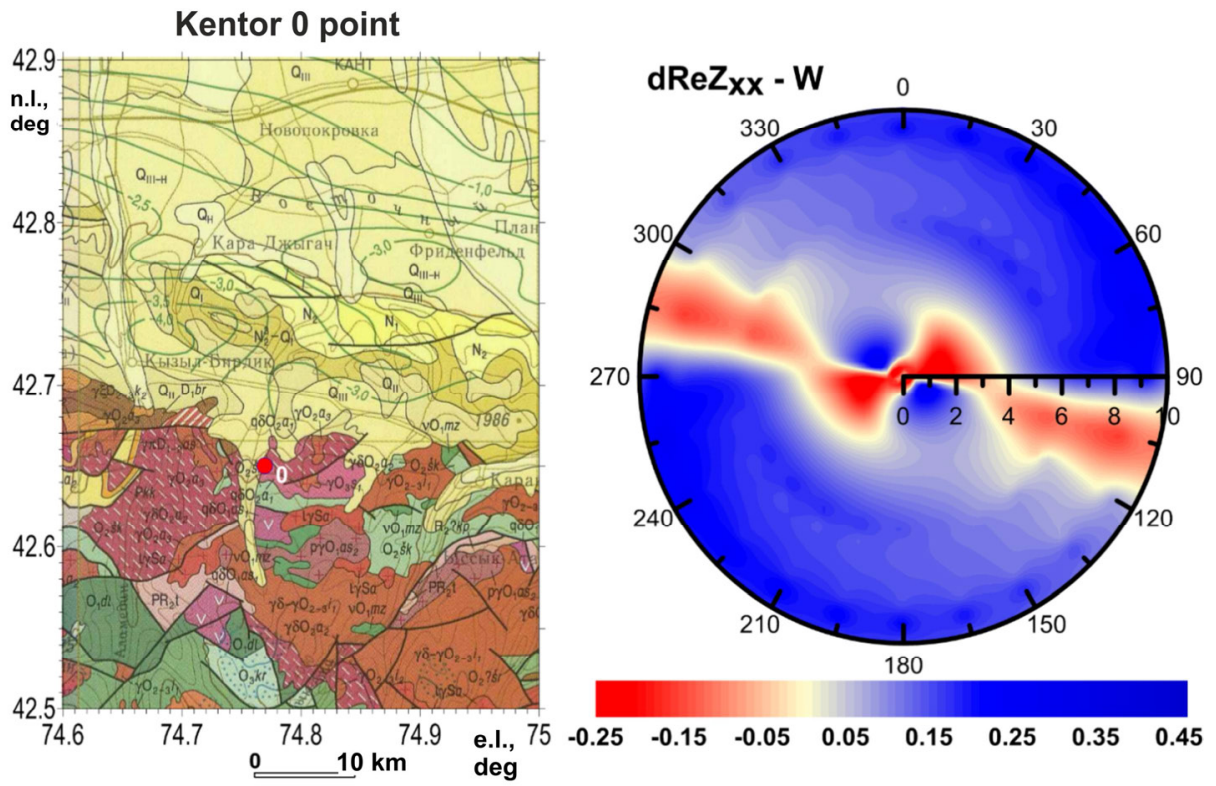

Fig. 6. Panel on the left - a fragment of the geological map near the location of the MT-monitoring mode station Kentor (0); the panel on the right - the distribution of the correlation coefficient (the Wiese vector $(\mathrm{W})$ and the vertical component of tidal forces $\mathrm{An}$ ) in polar coordinates, where the $\sqrt{T}_{\mathrm{T}}$ (T-period) is shown along the radius axis. Color and contour lines show the values of the correlation coefficient.

The data of azimuthal MT-monitoring of electromagnetic parameters are presented in the form of time-frequency series of apparent resistance, phase of impedance, real and imaginary parts of the main and additional impedances, as well as a tipper (Ro, $\phi$, ReZxy, ImZxy, ReZxx, ImZxx, W) (Figs. 4,5), which allows evaluate the variations in the electrical characteristics of the environment in the vicinity of the monitoring point. The reliability control of the RTC azimuthal MT monitoring is carried out by comparing and analyzing various types of data with each other and in correlation with tidal effects. To assess the tensosensitivity of active fault structures, correlation diagrams of the following time series were considered: variations in apparent resistivity, impedance phases, components of the impedance tensor (ReZxx, ImZxx, ReZxy, ImZxy) in comparison with the time series of lunar-solar tides (Figs. 5-6). The result of the comparison leads to a clear relationship between the parameters of the geomedium, depending on the distance and magnitude of the earthquake, as well as on the place of observation [11]. At the same time, the Ukok MT monitoring point (Fig. 3) is ideal for using it as a remote base, since it has a very low level of interference, and, therefore, allows obtaining high-quality data, while its location is interesting both from a geological point of view and for studying the behavior of electromagnetic parameters, as shown in Figure 5.

The results of experimental observations indicate a significant correlation between the variations of the vertical component of the lunar-solar tides and the electromagnetic field, which confirms the presence of cause-and-effect relationships between them. The results of the analysis of the cause-and-effect relationships between variations in electromagnetic parameters and lunar-solar tides are very important in solving the fundamental problem associated with the establishment of mechanisms for transforming geophysical fields. 


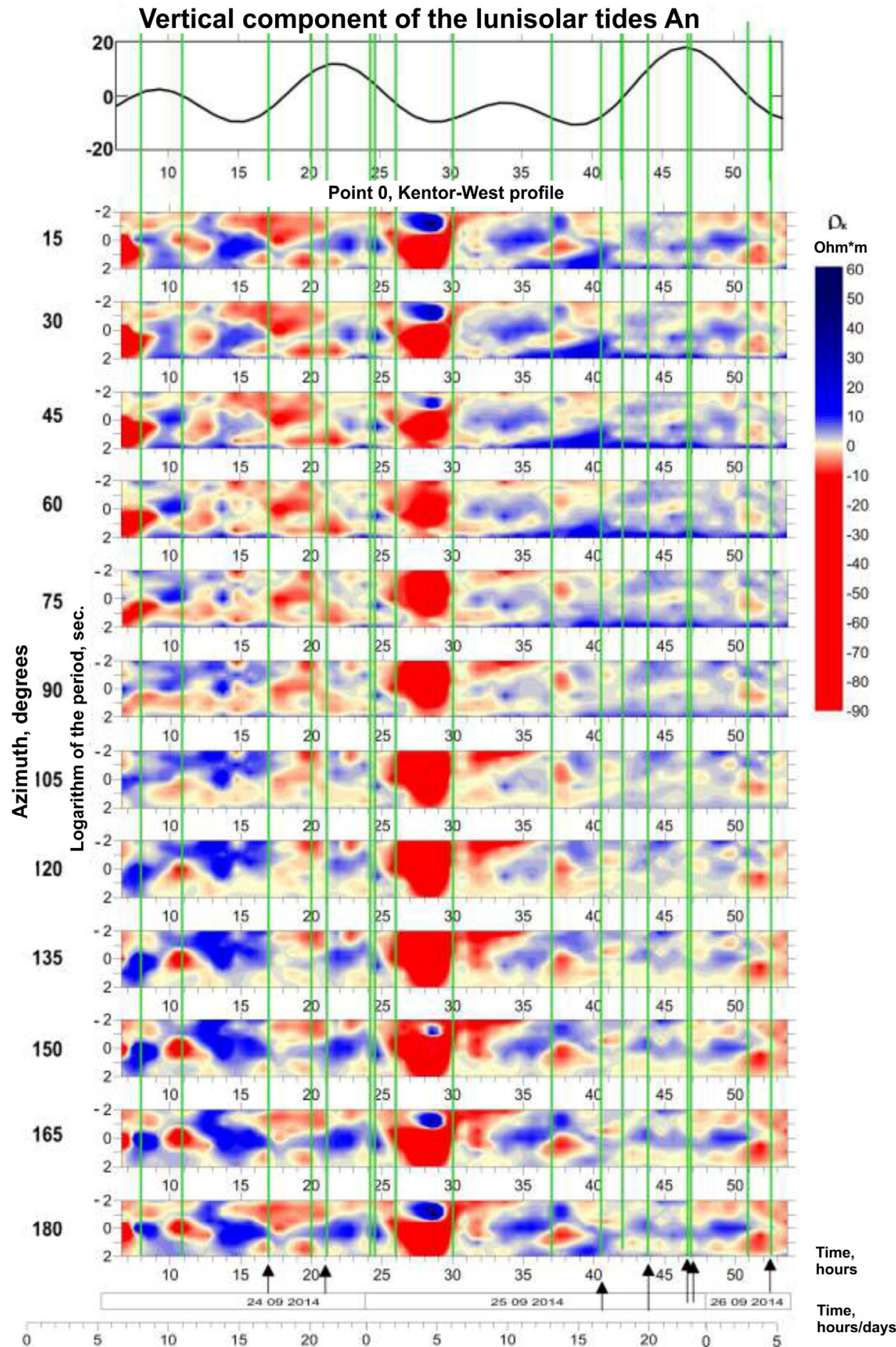

Fig 7. Time-frequency series of azimuthal MT-monitoring according to the data of regime point 0 of the Kentor minipolygon in comparison with seismicity. 


\section{Vertical component of the lunisolar tides An}

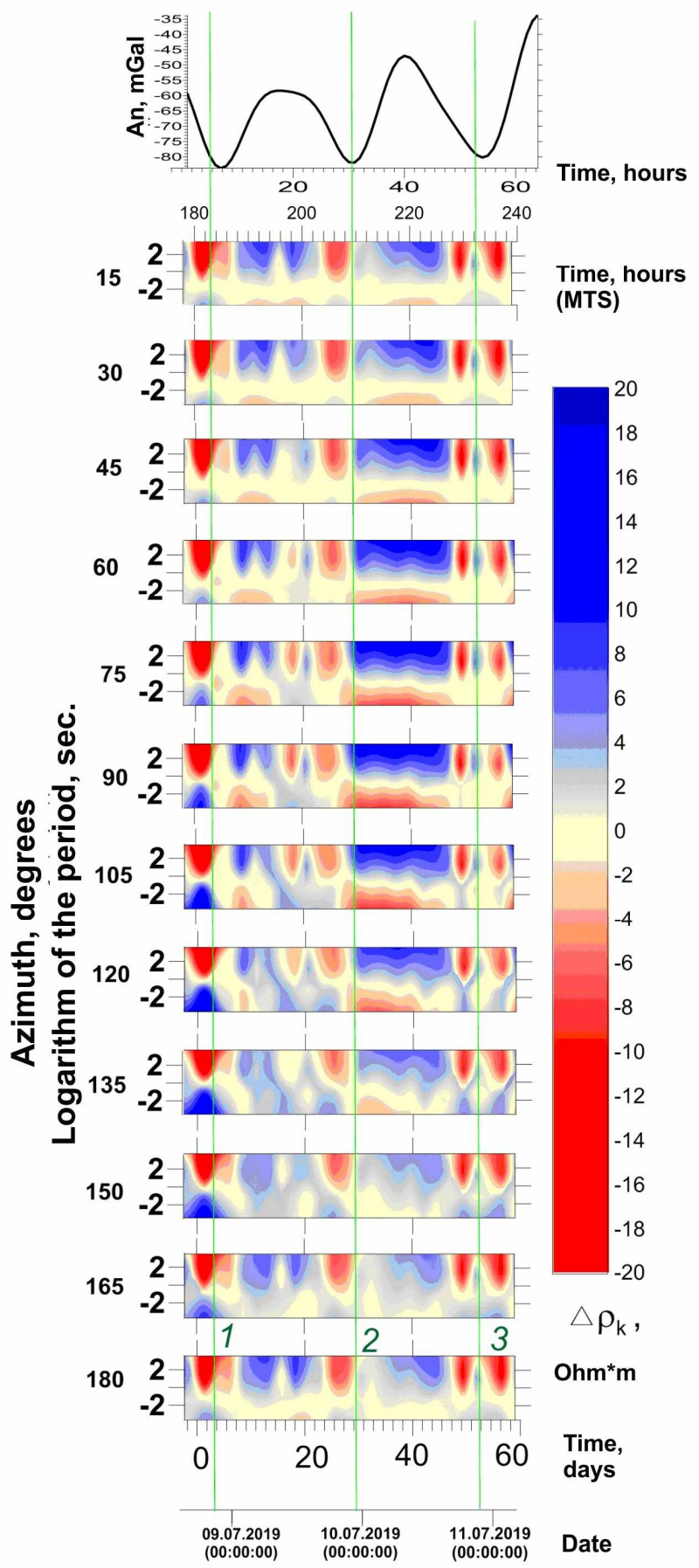

Fig. 8. The result of observations at Ukok-2. The upper graph is the vertical component of the lunarsolar tides, the lower sections are the MTS time-frequency series plotted with a $15^{\circ}$ step. Green lines with numbers show the time of registration of earthquakes. 
In addition, the determination of the relationship between variations in the electromagnetic parameters and the vertical component of lunar-solar tides opens up broad prospects for detecting and studying the dynamics of the development of fault structures and determining the degree of modern activity of their zones of dynamic influence, for improving existing and for developing new approaches to control the stress-strain state of the earth. crust of seismically active regions.

\section{Conclusions}

Based on the analysis of the time-frequency series of apparent resistivity variations in 12 azimuth directions for stationary MT monitoring stations Aksu and Chon-Kurchak for the relationship with lunar-solar tidal effects and seismic events that occurred during the period from 09 to 31 October 2018, the following conclusions can be drawn:

- the most important point is the picture of the change in the sign of variations on the pseudo-sections of the TFS;

- there is a fact of confinement of seismic events to areas of the TFS with high gradients of electromagnetic parameters, i.e. time intervals when, according to the concept of fluid redistribution, there is a significant change in the stress-strain state of rock massifs in the upper part of the Earth's crust;

- the analysis of the cross-correlation function indicates that the reason for the change in the parameters of the electromagnetic field can be lunar-solar tides.

For the Ukok-2 MT monitoring station, where experimental work was carried out in 2019, it can be noted that:

- there is a stable relationship between the anisotropy of electrical resistance, lunar-solar tidal effects and seismicity;

- the maximum amplitude of the vertical component by lunar-solar tidal effects corresponds to an increase in $\rho c$, a minimum - a decrease;

- the most clear variations of $\rho c$, to the gradient zones of which seismic events are confined, are expressed in the upper part of the section, that is, sharp changes in the stressstrain state occur at shallow depths, no more than $10 \mathrm{~km}$.

As a result of the analysis of the electromagnetic parameters of the environment for the relationship with lunar-solar tidal effects, it was found that in the overwhelming majority of cases, correlation dependences are most clearly manifested in changes in the real and imaginary parts of additional impedances than the main ones. Variations in the reactive component of electrical resistance (imaginary parts of impedances) associated with changes in the capacitive and inductive characteristics of rock mass during deformation processes respond to tidal effects and deformations no less actively than variations in the active component of electrical resistance (real parts of impedances). The results obtained contribute to a deeper understanding of the physics of the processes occurring in the earth's crust at different spatial-scale levels.

The work was carried out within the framework of the state assignment of the Reserch Station of the Russian Academy of Sciences on the topic AAAA-A19-119020190063-2.

\section{References}

1. S. Shlien, Geophys. J. Roy. Astron. Soc., 28 (27-34), (1972)

2. O. M. Barsukov, O. N. Sorokin, Izv. Phys. Solid Earth, 10 (100-102), (1973)

3. V.D. Bragin, A.M. Volykhin, Yu.A. Trapeznikov, Tectonophys, 202(2-4) (233-238) (1992) 
4. A.A. Zhamaletdinov, F.P. Mitrofanov, A.D. Tokarev, A.N. Shevtsov, Dokl. Earth. Sci., 371 (2), 403 (2000)

5. A.K. Saraev, M.I. Pertel, Z.M. Malkin, J. Appl. Geophys., 8(1-2), (2002)

6. T. Rikitake, 1, 4-26 (1972)

7. A.A. Spivak, Izv. Phys. Solid Earth, 46(4), 327 (2010)

8. A.A. Zhamaletdinov, A.N. Shevtsov, T.G. Korotkova, Izv. Phys. Solid Earth, 3(92105), (2018)

9. E.A. Bataleva, Dokl. Earth Sci., 468(1), 523 (2016)

10. E.A. Bataleva, V.Y. Batalev, A.K. Rybin. Izv. Phys. Solid Earth, 49(3), 402 (2013)

11. E.A. Bataleva, A.K. Rybin, V.E. Matiukov, Data, 4 (99) (2019) 